Ultrasound findings include unilateral effusions in 129 patients, bilateral in 18, and no effusion in 7 patients. Total 100 pleural procedures (diagnostic/therapeutic inspirations, chest drain insertion) were done under ultrasound guidance, and had only 1 complication of pneumothorax. This is thought possibly be due to 'trapped lung'. In 79 patients the effusion was small on chest x-ray, and out of which 41 patients we aspirated under guidance, but in 38 patients ( $48 \%$ of small effusions and $25 \%$ of all scans) the effusion was too small to be aspirated safely even under ultrasound guidance, so not intervened. $\mathrm{pH}$ of the pleural fluid ranged from 6.25-7.69. 16 patients had $\mathrm{pH}<7.2$, out of which 5 were empyema, 3 malignancy, and 3 rheumatoid. Pleural fluid cytology was positive for malignancy in 18 patients, and microbiology was positive in 6 patients. AAFB was negative in all patients.

During the last 6 months, 2 Consultants' and 3 Specialist Registrars' achieved level 1 competency and working towards level 2 . We have 3 half a day fixed sessions each week, and so far we have trained 8 junior level (include CT2, CT1, FY2, FY1) trainees who managed to attend our pleural sessions.

Conclusion Setting up a dedicated ultrasound guided pleural service vastly minimises the complications associated with pleural procedures, improves patient care, and provides great training opportunities for senior and junior level trainees. Our data strengthens the importance and safety of ultrasound guided pleural procedures.

\section{P120 THE USE OF IMAGE GUIDED CUTTING NEEDLE BIOPSY IN FAILED THORACOSCOPY: DIAGNOSTIC YIELD AND CORRELATION WITH CT RESULTS}

doi:10.1136/thoraxjnl-2012-202678.403

H Rostom, N Hassan, M Nagendran, E Brown, D Srikantharajah, M Maruthappu, A Manuel, NM Rahman. University Of Oxford, Oxford, United Kingdom

Introduction and Objectives Local anaesthetic thoracoscopy has a high diagnostic yield in undiagnosed pleural effusion, but cannot be performed in cases with heavily loculated pleural fluid or where lung is adherent to the chest wall. On table, ultrasound-guided trans-thoracic cutting needle biopsy provides a potential route to obtain tissue for histological analysis in such cases. There is currently a paucity of data addressing the diagnostic yield of this procedure when conducted by chest physicians in response to a failed thoracoscopic procedure. The present study was conducted to determine whether this method is able to obtain pleural tissue in cases of failed thoracoscopy, and whether the results correlated with the computed tomography (CT) scan results obtained before attempted thoracoscopy.

Methods Retrospective review of cases of unsuccessful thoracoscopy, which were converted to real-time ultrasound-guided cutting needle biopsy in the Pleural Unit between January 2010 and April 2012. The available histological results were assessed for the yield of pleural tissue, of definitive pleural diagnosis and compared to the pre-thoracoscopy CT scan results.

Results Of 147 attempted thoracoscopies, 11 (7.5\%) were not successful and were converted on table (using the same anaesthetised tract used to attempt to introduce the thoracoscope ports) to realtime image-guided trans-thoracic cutting needle biopsy. Of these, $1 / 11(9.1 \%)$ demonstrated pleural malignancy on histology (despite negative cytology), while in $8 / 11(72.7 \%)$ of cases, a benign pleural diagnosis was made. Pre-procedure CT scans were performed on 8 of the 9 patients for which a histological diagnosis was made, and were reported as likely malignant effusion in $4(50 \%)$ cases. $2 / 11(18.2 \%)$ biopsies failed to obtain sufficient tissue for histological diagnosis.

Conclusion Around $7.5 \%$ of attempted thoracoscopies are unsuccessful, and in these cases, real-time ultrasound guided cutting needle pleural biopsy obtains pleural tissue successfully in a high proportion. This approach offers the advantage of obtaining pleural tissue in a single procedure where thoracoscopy has failed. The true sensitivity and specificity of this technique now requires further assessment, including comparison to long term outcomes of patients with benign pleural histology obtained using this technique.

\section{P121 AMBULATORY TREATMENT FOR PNEUMOTHORAX: A SYSTEMATIC REVIEW OF THE LITERATURE AND CASE FOR OUTPATIENT ORIENTATED CARE?}

doi:10.1136/thoraxjnl-2012-202678.404

${ }^{1} \mathrm{FJH}$ Brims, ${ }^{2} \mathrm{~N}$ Maskell. 'University College London, London, UK; ${ }^{2}$ University Hospitals Bristol NHS Foundation Trust, Bristol, UK

Introduction Spontaneous pneumothorax (SP) is broken down into primary (PSP: no known underlying lung disease), secondary (SSP: known lung disease) and non-spontaneous from trauma or iatrogenic pneumothorax (IP). PSP carries a very low mortality. Current recognised treatments include a conservative approach, needle aspiration, intercostal chest tube (ICT) and surgery. A Heimlich valve (HV) is a lightweight one-way valve specifically designed for the ambulatory treatment of pneumothorax when used with an intercostal catheter. This systematic review was designed to examine the evidence for the use of $\mathrm{HV}$ in the management of adults with SP or IP.

Methods A systematic review was undertaken across nine electronic databases for studies reporting the use of HV for SP or IP. Randomised trials, case control studies and case series were included, unrestricted by year of publication. Post-thoracic surgery and traumatic pneumothorax reports were excluded. All publications were independently scored by two reviewers (FB \& NM). Measures of interest included: the use of a HV alone to manage SP or IP, successful treatment as outpatient (OP), complications and financial data. Results Eighteen studies were included reporting on the use of HV in 1235 patients, comprising 992 SP (of which 413 were reported as PSP) and 243 IP. The overall quality of the reports was low with only 2 RCTs ( $n=80$ patients), 1 consecutive case series and 14 case reports. Table 1 presents a summary of the outcome measures. In some reports it was not possible to distinguish outcomes between pneumothorax type. There were highly variable protocols and treatment practise across the studies. No deaths were reported, complications included four haemothoraces and one chest wall haematoma. Three studies presented data with cost analysis, with OP HV vs. inpatient ICT cost ratios ranging from 1:3 to 1:5.

Conclusions High quality data to support the use of HV for ambulatory treatment of pneumothorax is sparse. There are, however, data on $>1100$ patients from case reports suggesting that the use of $\mathrm{HV}$ for SP and IP is safe, effective and may facilitate outpatient orientated treatment of pneumothorax. There is need for a carefully designed RCT to examine this further.

Abstract P121 Table 1 Outcomes for use of HV with pneumothorax from 18 studies.

\begin{tabular}{llc}
\hline Outcome Measure: & $\mathbf{n} / \mathbf{N}=$ & $\%$ \\
\hline Success with HV alone: & & \\
All cases & $1060 / 1235$ & 85.8 \\
As outpatient & $761 / 977$ & 77.9 \\
PSP & $344 / 413$ & 83.3 \\
SSP & $110 / 124$ & 88.7 \\
latrogenic pneumothorax (IP) & $237 / 243$ & 97.5 \\
Need for surgery (all HV cases) & $119 / 1181$ & 10.1 \\
Reoccurrence (all HV cases: 6-31 months follow up) & & $7-24 \%$ \\
\hline
\end{tabular}

